RESEARCH PAPER

\title{
Comparison of clinical effects of epidural levobupivacaine morphine versus bupivacaine morphine in dogs undergoing elective pelvic limb surgery
}

\author{
Ilaria Cerasoli ${ }^{\mathrm{a}, 1}$, Alexandru Tutunaru ${ }^{\mathrm{a}, 1}$, Alessia Cenani ${ }^{\mathrm{a}}$, Juan Ramirez ${ }^{\mathrm{a}}$, \\ Johann Detilleux $^{\mathrm{b}}$, Marc Balligand ${ }^{\mathrm{a}}$ \& Charlotte Sandersen ${ }^{\mathrm{a}}$ \\ ${ }^{a}$ Department of Clinical Sciences, Faculty of Veterinary Medicine, University of Liège, Liège, \\ Belgium \\ ${ }^{b}$ Department of Quantitative Genetics, Faculty of Veterinary Medicine, University of Liège, Liège, \\ Belgium
}

Correspondence: Charlotte Sandersen, Department of Clinical Sciences, Faculty of Veterinary Medicine, University of Liège, Boulevard de Colonster 20, B41, 4000, Liège, Belgium. Email: charlotte.sandersen@ulg.be

${ }^{1}$ These two authors are joint first authors.

\begin{abstract}
Objective To evaluate the efficacy, in terms of the amount of rescue analgesia required, and the clinical usefulness of epidural injection of morphine with bupivacaine or levobupivacaine for elective pelvic limb surgery in dogs during a 24-hour perioperative period.
\end{abstract}

Study design Prospective, blinded, randomized clinical study.

Animals A group of 26 dogs weighing $31.7 \pm$ 14.2 (mean \pm standard deviation) $\mathrm{kg}$ and aged 54 \pm 36 months.

Methods All dogs were premedicated with methadone intravenously $\left(0.2 \mathrm{mg} \mathrm{kg}{ }^{-1}\right)$ and anaesthesia induced with diazepam $\left(0.2 \mathrm{mg} \mathrm{kg}^{-1}\right)$ and propofol intravenously to effect. After induction of anaesthesia, dogs randomly received a lumbosacral epidural injection of morphine $0.1 \mathrm{mg} \mathrm{kg}^{-1}$ with either levobupivacaine $0.5 \%$ (1 $\mathrm{mg} \mathrm{kg}^{-1}$; group LevoBM) or bupivacaine $0.5 \%$ ( $1 \mathrm{mg} \mathrm{kg}^{-1}$; group BM). Cardiovascular, respiratory and temperature values were recorded during the intraand postoperative period. A visual analogue scale, subjective pain scale, sedation scale and the short form of the Glasgow pain scale were assessed every 6 hours after epidural injection during 24 hours. The ability to stand and walk, neurological deficits and other side effects were assessed at the same time points. The amount of rescue analgesia (sufentanil intraoperatively and methadone postoperatively) was recorded.

Results No statistically significant differences were found between groups for any of the recorded data, with the exception of the incidence of spontaneous urination and postoperative rescue analgesia requirement. In group LevoBM four dogs spontaneously urinated at recovery while none of the dogs in group BM did ( $p=0.03)$ and seven dogs of group LevoBM required postoperative rescue analgesia versus none of the dogs in the BM group $(p=0.005)$.

Conclusions and clinical relevance Epidural Lev$\mathrm{oBM}$ is a suitable alternative to BM in healthy dogs during elective pelvic limb surgery. Epidural BM produced more urinary retention but better pain control compared to the same concentration and dose of LevoBM in dogs.

Keywords bupivacaine, dogs, epidural, pelvic limb surgery, levobupivacaine.

\section{Introduction}

Lumbosacral epidural anaesthesia is a common regional anaesthetic technique for management of dogs undergoing pelvic limb surgery. In the human and veterinary literature, it is well documented that 
lumbosacral epidural anaesthesia is able to alleviate pain in the pelvic limbs (Hendrix et al. 1996; Torske \& Dyson 2000; Dyson 2008). It has been argued that epidural anaesthesia provides better postoperative analgesia than parenteral opioids in humans (Block et al. 2003). Correct pain management reduces the recovery time, decreases the risk for secondary infections and surgical complications and results in a faster return to normal activities (Yeager et al. 1987).

Bupivacaine and levobupivacaine are local anaesthetics that, when dosed at lower concentrations, provide differential sensory and motor neural blockade (Camorcia et al. 2007) which is the blocking of pain and temperature sensations (A- $\delta, C$ fibres) while preserving motor function (A- $\alpha$ fibres). Bupivacaine has a chiral centre and therefore exists as a 50:50 mixture of two enantiomers [(S(-) and $\mathrm{R}(+)]$, both of which have similar potency and duration of action (Foster \& Markham 2000). Bupivacaine is commonly used in clinical practice because it has a long duration of action and a concentration of $0.5 \%$ of bupivacaine results in an adequate sensory and motor blockade for surgical procedures in companion animals (Torske \& Dyson 2000).

Levobupivacaine is the $\mathrm{S}(-)$-enantiomer of bupivacaine and has comparable anaesthetic properties. Experimental and human studies have shown that levobupivacaine is less cardiotoxic than bupivacaine (De Rossi et al. 2011) but with higher protein binding (97\% versus $95 \%$ ). This means that $<3 \%$ of levobupivacaine is free in the plasma and available to act on other tissues, possibly causing less unwanted side effects (McLeod \& Burke 2001).

The synergism of action of local anaesthetics and opioids has been widely investigated and reported in literature (Torske \& Dyson 2000; Kona-Boun et al. 2006; Leone et al. 2008; Abelson et al. 2011) and the analgesia is generally superior to that induced by each class injected alone. The addition of opioids improves the quality of pain relief without affecting the degree of motor blockade (Leone et al. 2008) and increases the duration. Bupivacaine-morphine is one of the most commonly used epidural drug combinations in small animal practice (Odette \& Smith 2013).

Although one study has compared the epidural effects of bupivacaine and levobupivacaine in conscious experimental dogs (Gomez de Segura et al. 2009), the effects of epidural levobupivacaine and morphine versus those of epidural bupivacaine and morphine have not been well studied.
The primary aim of this study was to evaluate the analgesia requirements and side effects of two epidural anaesthesia protocols in dogs undergoing elective pelvic limb surgery intraoperatively and for 24 hours postoperatively.

\section{Material and methods}

\section{Animals}

This study was conducted after institutional approval for animal experimentation (Commission d'éthique animale; 1237) and after obtaining informed consent from the animals' owners.

A power analysis was performed with $G$ power software (Faul et al. 2007) considering that values will be compared between both groups at each of the five time points ( $n=10$ classes). The power of the study was over $80 \%$ to detect an effect size of 0.37 (two-way ANOVA model) or 0.35 (chi-square test) in the scores with $\alpha=0.05$. By convention, these effect sizes are considered between medium and large effect. Therefore 13 animals were recruited per group.

Dogs undergoing elective tibial plateau advancement in the Veterinary Hospital of the University of Liège, Belgium were recruited for this prospective clinical study. No breed, sex, size, weight or age restrictions were imposed. A physical examination, complete blood cell count and serum biochemical analysis was performed on potential recruits. Dogs were excluded for presence of dermatitis in the area of the L7 to S1 intervertebral space, pelvic deformity, obesity (which precluded the palpation of the anatomical landmarks), aggression (which rendered them unable to participate in postoperative pain scoring) or low platelet count. All dogs were required to remain in the hospital for at least 24 hours after surgery.

\section{Study protocol}

The dogs were randomly allocated to one of the two groups by an external clinician using the Microsoft Excel (Microsoft Operations Ltd, Dublin, Ireland) random function. Anaesthetists, surgeons and both pain evaluators were unaware of group allocation. The treatment consisted of either $1 \mathrm{mg} \mathrm{kg}^{-1}$ bupivacaine (Marcaine; AstraZeneca, Belgium) and 0.1 $\mathrm{mg} \mathrm{kg}^{-1}$ of preservative-free morphine sulphate $1 \%$ (Morphine HCL Sterop; Sterop, Belgium) (group BM) or $1 \mathrm{mg} \mathrm{kg}^{-1}$ levobupivacaine $0.5 \%$ (Chirocaine; AbbVie, Belgium) and $0.1 \mathrm{mg} \mathrm{kg}^{-1}$ morphine sulphate (group LevoBM). 
Food, but not water, was withheld for 12 hours before anaesthesia. On the day of surgery, a catheter was placed aseptically into the left or right cephalic vein.

Preoperative baseline values were taken for heart rate $(\mathrm{HR})$ and respiratory rate $\left(f_{\mathrm{R}}\right)$ and systolic arterial blood pressure (SAP) was measured by the use of Doppler (Model 811-B; Parks Medical, NV, USA) before premedication. Dogs were then premedicated with $0.2 \mathrm{mg} \mathrm{kg}^{-1}$ of methadone (Mephenon; Denolin, Belgium) intravenously (IV), followed by induction with $0.2 \mathrm{mg} \mathrm{kg}^{-1}$ diazepam (Valium; Roche, Belgium) and propofol (Diprivan 1\%, Astra Zeneca, Belgium) IV to effect. The trachea was intubated with an appropriately sized endotracheal tube and connected to a small animal anaesthesia re-breathing circuit. Anaesthesia was maintained with isoflurane (IsoFlo, Abbott Laboratories Ltd, UK) in 1:1 oxygen/ air mixture. Continuous mechanical ventilation (Cicero; Dräger, Germany) was provided and the minute volume was adjusted to maintain the endtidal carbon dioxide $\left(\mathrm{PE}^{\prime} \mathrm{CO}_{2}\right)$ between 35 and 45 $\mathrm{mmHg}(4.6-5.9 \mathrm{kPa})$. The end-tidal isoflurane (FE'Iso) was kept between 1.1 and $1.3 \%$.

The dogs received $10 \mathrm{~mL} \mathrm{~kg}^{-1}$ hour $^{-1}$ Hartmann's solution IV throughout the surgical procedure and $20 \mathrm{mg} \mathrm{kg}^{-1}$ cefazoline IV 30 minutes prior to surgical incision and every 2 hours during the surgery.

After induction, physiological and anaesthetic parameters were monitored via multiparametric monitors (Datex Cardiocap II, CH-2S-23-02, Datex Capnomac Ultima ULT V-23-00; Acertys, Belgium) and included electrocardiography, pulse oximetry, body temperature, $\mathrm{PE}^{\prime} \mathrm{CO}_{2}$, and $\mathrm{FE}^{\prime}$ Iso.

Dogs were then positioned in sternal recumbency and the lumbosacral area clipped and prepared aseptically for the epidural injection. A 22 gauge Tuohy needle was introduced into the lumbosacral epidural space. Correct epidural placement was confirmed by the hanging drop test and loss of resistance method using a test injection of sterile $\mathrm{NaCl}$ $0.9 \%$ at room temperature with a low resistance syringe (Perifix; B. Braun, Germany). The patient was excluded from the study if blood was seen at the end of the needle or after three unsuccessful attempts to introduce the needle in the epidural space. Then, $0.2 \mathrm{~mL} \mathrm{~kg}^{-1}$ of the assigned epidural treatment was injected over 90 seconds with a continuous evaluation of resistance to injection to detect possible displacement of the needle. Dogs were positioned in right or left lateral recumbency for at least 5 minutes after the epidural injection to maximize drugs contact time on the nerve roots involved in the surgical procedure (Torske \& Dyson 2000).

Hypotension was defined as a Doppler measured systolic arterial pressure (SAP) below $90 \mathrm{mmHg}$ and treated by lowering the $\mathrm{FE}^{\prime}$ Iso (minimum 1.1\%). In the event of bradycardia (considered as a HR 15\% lower than baseline) $5 \mu \mathrm{g} \mathrm{kg}^{-1}$ glycopyrrolate (Robinul-V; Fort Dodge, IA, USA) was administered IV and repeated after 10 minutes if required. If these measures were unsuccessful, a bolus of crystalloids $(10 \mathrm{~mL}$ $\mathrm{kg}^{-1}$ over 10 minutes) or colloids ( $3 \mathrm{~mL} \mathrm{~kg}^{-1}$ over 10 minutes) was administered. When hypotension still persisted, dobutamine was administered at an initial rate of $5 \mu \mathrm{g} \mathrm{kg}^{-1}$ minute ${ }^{-1} \mathrm{IV}$. Body temperature was maintained between $37.0^{\circ} \mathrm{C}$ and $38.5^{\circ} \mathrm{C}$ using warm air blankets (Mistral; TSC, The Netherlands).

Rescue analgesia consisting of sufentanil (Sufenta; Janssen Cilag, Belgium; $0.2 \mu \mathrm{g} \mathrm{kg}^{-1}$ bolus, $0.5 \mu \mathrm{g}$ $\mathrm{kg}^{-1}$ hour $^{-1}$ continuous infusion adjusted on demand) was administered during surgery when HR, $f R$ or SAP increased by $>20 \%$ of the baseline values recorded before epidural injection. Propofol 1-2 mg $\mathrm{kg}^{-1}$ IV was administered in the event of gross purposeful movements. Carprofen $4 \mathrm{mg} \mathrm{kg}^{-1}$ (Rimadyl solution; Zoetis, Belgium) was administered subcutaneously at the end of the procedure.

Manual expression of the bladder was only performed when the bladder was considered too distended. The dogs were moved to the recovery area and the trachea extubated once the dogs were able to swallow.

Postoperatively, two trained observers assessed the degree of pain in each dog using the short form of the Glasgow composites measures pain score (from $0=$ no pain to $24=$ severe pain), a visual analogue scale (VAS; from $0 \mathrm{~cm}=$ no pain to $10 \mathrm{~cm}=$ severe pain), a subjective pain score (non, mild, moderate, severe) and a sedation score (from $0=$ fully awake to $4=$ asleep, unable to raise head) according to scores used by Murrell et al. (2008). Details can be found in Table S1. The mean of the scores of the two evaluators were considered for the analysis of the results. Practically, the dog's behaviour and VAS were assessed initially from outside the kennel and any spontaneous vocalisation, attention to the surgical area and posture were recorded. After this, the door of the kennel was opened and the dog was called to encourage movement. The dog was taken out from the cage and walked. The neurological deficit was assessed by testing the righting reflex, and the ability to stand and walk which were all graded as normal or not normal. Details can be found in Table S2. Gentle 
pressure was applied around the wound and the dog's response to touch was assessed. Finally, an overall impression of the observer about the comfort and demeanour was recorded.

All the assessments and the presence or absence of other side effects such as respiratory depression, sedation, nausea (hypersalivation, retching, excessive licking, and chewing), vomiting, appetite, pruritus and hypotension were recorded at the time of extubation (recovery time) and every 6 hours after the epidural injection (timepoints 6, 12,18, and 24). Methadone $0.2 \mathrm{mg} \mathrm{kg}^{-1}$ was administered as rescue analgesia if the subjective pain scale was severe or if the Glasgow pain score was above 6 out of a possible 24. If the Glasgow pain score was above 6 while the subjective pain score was assessed as none or mild pain $0.1 \mathrm{mg} \mathrm{kg}^{-1}$ methadone IV was given.

A tranquillizer $\left(0.01 \mathrm{mg} \mathrm{kg}^{-1}\right.$ acepromazine IV; Placivet; Kela Lab, Belgium) was prepared if dysphoria was suspected and a second evaluation was performed 30 minutes later. Palpation of the bladder was performed every 6 hours, and in case of excessive distension, an external manual expression was performed and recorded.

\section{Statistical analysis}

Normality of the data was assessed with the univariate procedure (SAS System 9.3; Institute Inc., NC, USA). Data that were not normally distributed $\left(f_{\mathrm{R}}\right.$, temperature and subjective pain scale values) were normalized with the use of Boxcox and then analysed. An ANOVA test was used for continuous parameters and logistic regression for binary variables. A chi-square test was used for the incidence of postoperative rescue analgesia. This was necessary, as the logistic regression could not analyse the group were the incidence was equal to zero. Overall $p$ values $<0.05$ were considered significant. Results are presented as mean \pm standard deviation and median (range).

\section{Results}

Of the 30 dogs initially recruited, four dogs were excluded: one because of difficult behaviour and three because of obesity. All of the 26 included dogs completed the study. There were no significant differences between groups with respect to age, weight, baseline physiological parameters and duration of anaesthesia and surgery. The dogs weighed $31.7 \pm$ $14.2 \mathrm{~kg}$ and had an age of $54 \pm 36$ months.

\section{Intraoperative cardiovascular and respiratory variables}

There were no significant differences between groups LevoBM and BM for HR, SAP, $\mathrm{PE}^{\prime} \mathrm{CO}_{2}$ values, $f \mathrm{R}$ and temperature (Table 1).

\section{Intraoperative rescue analgesia}

In each group, two of 13 dogs needed sufentanil rescue analgesia during surgery. Rescue analgesia was required at 60 and 190 minutes after epidural injection in group BM and at 160 and 235 minutes after epidural injection in group LevoBM.

\section{Intraoperative cardiovascular support}

During surgery, four of 13 (31\%) dogs in group BM needed cardiovascular support. Glycopyrrolate was administered to two of the dogs and a fluid bolus to the other two dogs. In group LevoBM, seven of 13 (54\%) dogs required cardiovascular support. Glycopyrrolate was administered to five of 13 (39\%) dogs and fluid boluses to four of $13(30.7 \%)$ dogs. Two dogs required both glycopyrrolate and fluid boluses. None of the dogs required a dobutamine infusion.

\section{Postoperative assessment}

The number of dogs experiencing nausea is displayed in Table 2. One nausea episode in group BM was

Table 1 Intraoperative variables during general anaesthesia of dogs undergoing pelvic limb surgery and receiving either epidural bupivacaine-morphine (Group BM) or levobupivacaine-morphine (Group LevoBM)

\begin{tabular}{|c|c|c|c|c|c|c|}
\hline & & Variable & & & & \\
\hline Group & $n$ & $\begin{array}{l}\text { HR } \\
\text { (beats minute }^{-1} \text { ) }\end{array}$ & $\mathrm{SAP}(\mathrm{mmHg})$ & $\begin{array}{l}f_{\mathrm{R}} \\
\text { (breaths minute }^{-1} \text { ) }\end{array}$ & $\begin{array}{l}\mathrm{PE}^{\prime} \mathrm{CO}_{2} \\
{[\mathrm{mmHg}(\mathrm{kPa})]}\end{array}$ & $\begin{array}{l}\text { Temperature } \\
\left({ }^{\circ} \mathrm{C}\right)\end{array}$ \\
\hline BM & 13 & $82 \pm 19$ & $110 \pm 12$ & $18 \pm 2$ & $\begin{array}{l}39 \pm 3 \\
(5.2 \pm 0.4)\end{array}$ & $36.7 \pm 0.6$ \\
\hline LevoBM & 13 & $84 \pm 15$ & $114 \pm 21$ & $15 \pm 4$ & $\begin{array}{l}40 \pm 4 \\
(5.3 \pm 5.3)\end{array}$ & $37.1 \pm 0.5$ \\
\hline
\end{tabular}

$f_{\mathrm{R}}$, respiratory rate; $\mathrm{HR}$, heart rate; $\mathrm{PE}^{\prime} \mathrm{CO}_{2}$, end-tidal carbon dioxide concentration; SAP, systolic arterial pressure.

340 (c) 2017 Association of Veterinary Anaesthetists and American College of Veterinary Anesthesia and Analgesia. Published by Elsevier Ltd. All rights reserved., 44, 337-345 
Table 2 Number of dogs requiring postoperative rescue analgesia and experiencing postoperative neurological deficits, nausea, defecation and number of animals able to stand, walk and spontaneously empty their bladder (cumulative data) after undergoing pelvic limb surgery and receiving either epidural bupivacaine-morphine (Group BM, $n=13$ ) or levobupivacaine-morphine (Group LevoBM, $n=13$ )

\begin{tabular}{|c|c|c|c|c|c|c|}
\hline \multirow[b]{2}{*}{ Parameter } & \multirow[b]{2}{*}{ Group } & \multicolumn{5}{|c|}{ Time point* } \\
\hline & & $\mathrm{R}$ & 6 & 12 & 18 & 24 \\
\hline \multirow[t]{2}{*}{ Rescue analgesia $(n)$} & $\mathrm{BM}$ & & 0 & 0 & 0 & 0 \\
\hline & LevoBM & & 5 & 4 & 6 & 3 \\
\hline \multirow[t]{2}{*}{ Neurological deficits $(n)$} & $\mathrm{BM}$ & 2 & 1 & 0 & 0 & 0 \\
\hline & LevoBM & 0 & 0 & 0 & 0 & 0 \\
\hline \multirow[t]{2}{*}{ Defecation $(n)$} & $\mathrm{BM}$ & 10 & 2 & 2 & 0 & 1 \\
\hline & LevoBM & 6 & 6 & 1 & 1 & 2 \\
\hline \multirow[t]{2}{*}{ Nausea $(n)$} & $\mathrm{BM}$ & 0 & 0 & 1 & 0 & 0 \\
\hline & LevoBM & 1 & 2 & 4 & 6 & 3 \\
\hline \multirow[t]{2}{*}{ Standing (cumulative data) $(n)$} & BM & 0 & 5 & 10 & 11 & 13 \\
\hline & LevoBM & 1 & 10 & 12 & 13 & 13 \\
\hline \multirow[t]{2}{*}{ Able to walk (cumulative data) $(n)$} & $\mathrm{BM}$ & 0 & 4 & 10 & 11 & 13 \\
\hline & LevoBM & 1 & 9 & 12 & 13 & 13 \\
\hline \multirow[t]{2}{*}{ Urination (cumulative data) ( $n$ ) } & $\mathrm{BM}$ & 0 & 2 & 8 & 8 & 11 \\
\hline & LevoBM & 4 & 5 & 8 & 9 & 13 \\
\hline
\end{tabular}

${ }^{*}$ Recovery $(\mathrm{R})$ indicates the extubation time point. Time points 6, 12, 18 and 24 are 6, 12, 18 and 24 hours after epidural injection, respectively. Rescue analgesia consisted of $0.1 \mathrm{mg} \mathrm{kg}^{-1}$ methadone administered intravenously.

correlated with an episode of vomiting. In the LevoBM group, dogs showed nausea at different time points but no vomiting (Table 2). The difference between the groups was not statistically significant. Four of the 16 nausea episodes were related to the administration of methadone.

None of the dogs were able to urinate spontaneously in the BM group at recovery ( $p=0.03$; Table 2$)$. Uncontrolled urinary outflow was observed in one dog in the BM group.

The incidence of defecation was similar between groups (Table 2) and neither hypotension nor pruritus were observed during the postoperative period.

Very few animals experienced neurological deficits during recovery but one dog continued to manifest deficits 6 hours after epidural injection (Table 2).

Although there seemed to be a slightly higher proportion of dogs that were able to walk and stand within 6 hours after epidural injection in group LevoBM, there were no statistically significant differences found between groups (Table 2). The ability to walk was regained completely 18 and 24 hours after epidural injection groups LevoBM and BM, respectively.

\section{Postoperative analgesia}

There were no significant differences between groups in the sedation and pain scores (Table 3). None of the dogs in group BM needed rescue analgesia during the first 24 hours postsurgery $(p=0.005)$ and of the seven of dogs in the LevoBM group requiring analgesia, four needed one or two doses of a low dose of methadone $0.1 \mathrm{mg} \mathrm{kg}^{-1}$ while the other three received three doses of rescue analgesia.

\section{Discussion}

There were no statistically significant differences between groups for any of the recorded data, with the exception of the incidence of spontaneous urination at recovery and postoperative rescue analgesia requirement. In group LevoBM four dogs spontaneously urinated at recovery while none of the dogs in group BM did $(p=0.03)$ and seven dogs of group LevoBM required postoperative rescue analgesia versus none of the dogs in the BM group ( $p=0.005$ ). Overall, the difference between groups appear to be minimal and both bupivacaine plus morphine and levobupivacaine plus morphine seem to be acceptable options for epidural anaesthesia in healthy dogs undergoing elective orthopaedic procedures. The small number of dogs included in this study might have precluded us from detecting rare events in either group.

There were no differences in the intraoperative cardiovascular effects between groups. The intraoperative analgesic effects were also similar in both groups. In each group two dogs required intraoperative rescue analgesia, which could be 
Table 3 Median (range) values for subjective sedation and pain scale and for visual analogue scale (VAS) and Glasgow pain scale scores in animals from group bupivacaine-morphine (Group BM, $n=13$ ) and levobupivacaine-morphine (Group LevoBM, $n=13$ ) at different time points*

\begin{tabular}{llll}
\hline & Time point & Group BM & Group LevoBM \\
\hline Subjective sedation score & $\mathrm{R}$ & $2(1-2)$ & $0(0-4)$ \\
& 6 & $1.5(0-2)$ & $1(0-2)$ \\
& 12 & $0(0-4)$ & $1(0-2.5)$ \\
& 18 & $0(0-1.5)$ & $1(0-3)$ \\
Subjective pain scale score & 24 & $0(0-0.5)$ & $0(0-1)$ \\
& $\mathrm{R}$ & $2(1-2)$ & $1(1-2)$ \\
& 6 & $1.5(1-2)$ & $1.5(1.5-4)$ \\
& 12 & $1(1-2.5)$ & $1(1-2)$ \\
Glasgow pain scale score & 18 & $1(1-2)$ & $1(1-2.5)$ \\
& 24 & $1(1-1.5)$ & $1(1-2)$ \\
& $\mathrm{R}$ & $3.5(1-6)$ & $0(0-4)$ \\
& 6 & $3(1-6)$ & $4.5(1-9.5)$ \\
& 12 & $1(0-6)$ & $2.5(1-8)$ \\
& 18 & $1(0-3.5)$ & $4(1-6.5)$ \\
& 24 & $0.5(0-3.5)$ & $1.5(0-1.5)$ \\
& $\mathrm{R}$ & & $1.4(0.1-4)$ \\
& 6 & $0.7(0.15-2.1)$ & $0.75(0.1-2.5)$ \\
& 12 & $0.5(0.05-1.2)$ & $0.9(0.15-2.05)$ \\
& 18 & $0.5(0-0.85)$ & $0.8(0.1-1.3)$ \\
\hline
\end{tabular}

${ }^{*}$ Recovery $(R)$ indicates the extubation time point, time points $6,12,18,24$ indicate from after the recovery to the $6^{\text {th }}$, from the $7^{\text {th }}$ to the $12^{\text {nd }}$, from the $13^{\text {th }}$ to the $18^{\text {th }}$ and from the $19^{\text {th }}$ to the $24^{\text {th }}$ hours after epidural injection, respectively.

considered as partial or total failure of epidural anaesthesia in four of $26 \mathrm{dogs}$ (15\%). The need for intraoperative rescue analgesia was relatively low and may be partially explained by nonuniform spread of the drugs in the epidural space (Reina et al. 2009). A $2-5 \%$ epidural failure rate has been reported in human medicine (Ranasinghe et al. 2003). Cox et al (1998) compared epidural bupivacaine $0.5 \%$ and levobupivacaine $0.5 \%$, administered at the same dose, for lower limb surgery in humans and found no clinical differences regarding the onset time, duration of action and spread of intensity of the sensory block.

Our study revealed a significant difference $(p=$ 0.005 ) in the need for postoperative rescue analgesia between the two drugs that were used at the same concentration $(0.5 \%)$ and volume (total of $0.2 \mathrm{~mL}$ $\mathrm{kg}^{-1}$ ). Group BM did not require extra analgesia postoperatively, while in the LevoBM group, rescue analgesia was required in seven of the 13 dogs. In both human and veterinary literature, the analgesic effect of levobupivacaine is indeed considered less and of shorter duration compared to the racemic mixture (Camorcia et al. 2007; Gomez de Segura et al. 2009). However, four of the seven dogs requiring postoperative rescue analgesia, received a very low dose of $0.1 \mathrm{mg} \mathrm{kg}^{-1}$ and most of the time this dose was administered once. Further, there were no statistically significant differences for the pain scores between the groups.

Assessment of pain in animals is far from being objective and lacks any gold standard compared to humans, where the gold standard of pain evaluation is self-reporting (Mathew \& Mathew 2003). Simple unidimensional pain scales have been proven unreliable for pain assessment in dogs (Holton et al. 1998). A newer study evaluated the modified Glasgow pain scale by comparing the results with a simple descriptive pain scale and found it reliable in assessing acute pain in dogs (Murrell et al. 2008). In our study, in order to limit subjectivity, pain was assessed by two trained observers, unaware of treatment allocation, using three different pain scales.

\section{Motor block}

Contradictory results regarding the duration of epidural anaesthesia in humans are reported in literature. A longer duration of motor block has been described with levobupivacaine than with bupivacaine (Foster \& Markham 2000). In our study, a 
shorter duration of action was recorded. This is in agreement with the study of Gomez de Segura et al. (2009), which showed that levobupivacaine, at a concentration of $0.5 \%$, caused a shorter loss of motor function of $183 \pm 99$ minutes, while bupivacaine caused a loss of motor function of $238 \pm 103$ minutes. In our study, $70 \%$ of the dogs in group LevoBM and $46 \%$ of the dogs in group BM were able to stand within 6 hours after the epidural injection. A complete resolution of the motor block (no ataxia, full coordination of the hind limbs) in $100 \%$ of the dogs was recorded at 18 and 24 hours after epidural injection, for groups LevoBM and BM, respectively. The duration of the motor block was longer than expected with either drug. Possibly, morphine potentiated the effects of bupivacaine and levobupivacaine in the epidural space. To the authors' knowledge, local anaesthetic and opioid synergism has been described for the analgesic effect of epidural anaesthesia, but not for the motor block. Importantly, the ability to walk was also influenced by the bandage applied to the affected limb and therefore not always related to a real lack of motor activity. In fact, only three dogs showed mild neurological deficit during the postoperative period (two of 13 group BM dogs during recovery and 6 hours after epidural injection and one of 13 dogs in group LevoBM 6 hours after the epidural injection).

Delayed hair regrowth, pruritus and urinary retention are known complications of epidural anaesthesia in dogs (Kalchofner Guerrero et al. 2014). Urinary retention after epidural anaesthesia in dogs has been described with an incidence of 3-44\% (Campoy et al. 2012; Troncy et al. 2002). In human medicine, the incidence of urinary retention is considered to be dose-related and is estimated to be between 15 and $100 \%$. In the case of opioids, the mechanism is likely to be related to interaction with the opioid receptors in the sacral part of the spinal cord, the suppression of detrusor muscle and the sensation to urinate (Rawal et al. 1983). According to the study of Rawal et al. (1983), the spontaneous recovery of bladder function after epidural morphine occurs in 14-16 hours in man. Canine cystometric studies showed a relaxation of the detrusor muscle and an increased bladder capacity following the spinal injection of opioids (Kalchofner Guerrero et al. 2014). Because of that, dogs required external bladder expression or urethral catheterization after the use of morphine in the epidural space. $\mu$-Agonists also tend to decrease the urine production by stimulation of the antidiuretic hormone. However, $\kappa$-agonist activity of morphine may outweigh this side effect by decreasing the production of the hormone (Mercadante \& Arcuri 2004).

Nausea (and vomiting) is one of the most common complications of general anaesthesia in humans. In the current study, one dog in group BM showed signs of nausea and vomited. By contrast, nausea was observed in six dogs in group LevoBM. As the difference between groups was not significant, it may have been an incidental finding as a result of the small sample size. To the authors' knowledge, a higher incidence of nausea after epidural administration of levobupivacaine compared to bupivacaine has indeed not been reported in veterinary or human literature. Although the group that received more often additional methadone in the postoperative period was also the group that showed a higher incidence of nausea, there does not seem to be a relationship between nausea and administration of methadone. In general, methadone causes nausea less often when compared to morphine (Monteiro et al. 2009).

The most common problem during the perioperative period was hypotension. Hypotension during epidural anaesthesia is normally secondary to sympathetic block, relative overdose of anaesthetic drugs and underlying unrecognized hypovolaemia (Odette \& Smith 2013). Furthermore, all dogs were mechanically ventilated to minimize the effect of a possible respiratory acidosis. This may have contributed to hypovolaemia related hypotension, through a decrease in preload and stroke volume (Hopper \& Powell 2013). In human medicine, low doses of isobaric levobupivacaine $0.5 \%$ have been described to produce less hypotension compared to hyperbaric bupivacaine 0.5\% (Herrera et al. 2014). Nevertheless, an incidence of hypotension related to the epidural use of levobupivacaine of $31 \%$ has been described (Foster \& Markham 2000). The small sample size could have influenced these results and a much larger number of animals needs to be examined in order to evaluate rare events.

The lack of statistically significant differences between the groups in this study suggests that levobupivacaine has minimal side effects when used for epidural anaesthesia in healthy dogs undergoing pelvic limb surgery. Due to the higher incidence of urinary retention in the LevoBM group, it is advised to assess the patient's ability to urinate for a period of 24 hours after epidural injection. 


\section{Conflict of interest}

Authors declare no conflict of interest.

\section{Authors' contributions}

IC, AT, AC, JD, MB and CS designed the study. AT, IC, $\mathrm{AC}, \mathrm{MB}$ and JR carried out all technical procedures and performed the data acquisition. JD performed all statistical analyses. IC, AT, JD, MB and CS wrote the manuscript. All authors read and approved the final manuscript.

\section{References}

Abelson AL, Armiage-Chan E, Lindsey JC et al. (2011) A comparison of epidural morphine with low dose bupivacaine versus epidural morphine alone and motor and respiratory function in dogs following splenectomy. Vet Anaesth Analg 38, 213-223.

Block BM, Liu SS, Rowlingson AJ et al. (2003) Efficacy of postoperative epidural analgesia: a meta-analysis. J Am Med Assoc 290, 2455-2463.

Camorcia M, Capogna G, Berritta C et al. (2007) The relative potencies for motor block after intrathecal ropivacaine, levobupivacaine, and bupivacaine. Anaesth Analg 104, 904-907.

Campoy L, Martin-Flores M, Ludders JW et al. (2012) Comparison of bupivacaine femoral and sciatic nerve block versus bupivacaine and morphine epidural for stifle surgery in dogs. Vet Anaesth Analg 39, 91-98.

Cox CR, Faccenda KA, Gilhooly C et al. (1998) Extradural S(-)-bupivacaine: comparison with racemic RS-bupivacaine. Br J Anaesth 80, 289-293.

De Rossi R, De Barros ALC, Silva-Neto AB et al. (2011) Hyaluronidase shortens levobupivacaine lumbosacral epidural anaesthesia in dogs. J Small Anim Pract 52, 195-199.

Dyson DH (2008) Perioperative management in veterinary patients. Vet Clin North Am Small Anim Pract 38, 1309-1327.

Faul F, Erdfelder E, Lang AG, Buchner A (2007) G*Power 3: a flexible statistical power analysis program for the social, behavioral, and biomedical sciences. Behav Res Methods 39, 175-191.

Foster RH, Markham A (2000) Levobupivacaine: a review of its pharmacology and use as a local anaesthetic. Drugs 59, 551-579.

Gomez de Segura IA, Menafro A, Garcia-Fenandez P et al. (2009) Analgesic and motor-blocking action of epidurally administered levobupivacaine or bupivacaine in the conscious dog. Vet Anaesth Analg 36, 485-494.

Hendrix PK, Raffe MR, Robinson EP et al. (1996) Epidural administration of bupivacaine, morphine, or their combination for postoperative analgesia in dogs. J Am Vet Med Assoc 209, 598-607.

Herrera R, De Andrés J, Estañ L et al. (2014) Hemodynamic impact of isobaric levobupivacaine versus hyperbaric bupivacaine for subarachnoid anesthesia in patients aged 65 and older undergoing hip surgery. BMC Anesthesiol 14, 97.

Holton LL, Scott EM, Nolan AM et al. (1998) Comparison of three methods used for assessment of pain in dogs. J Am Vet Med Assoc 212, 61-66.

Hopper K, Powell LL (2013) Basic of Mechanical ventilation for Dogs and Cats. Vet Clin North Am Small Anim Pract 43, 955-969.

Kalchofner Guerrero KS, Guerrero TS, SchweizerKölliker M et al. (2014) Incidence of delayed hair regrowth, pruritus, and urinary retention after epidural anaesthesia in dogs. Tierarztl Prax Ausg K Kleintiere Heimtiere 2, 94-100.

Kona-Boun JJ, Cuvelliez S, Troncy E (2006) Evaluation of epidural administration of morphine or morphine and bupivacaine for postoperative analgesia after premedication with opioid analgesic and orthopedic surgery in dogs. J Am Vet Med Assoc 229, 1103-1111.

Lascelles BD, Butterworth SJ, Waterman AE (1994) Postoperative analgesic and sedative effects of carprofen and pethidine in dogs. Vet Rec 134, 187-191.

Leone S, Di Cianni S, Casati A, Fanelli G (2008) Pharmacology, toxicology, and clinical use of a new long acting local anesthetics, ropivacaine and levobupivacaine. Acta Biomed 79, 92-105.

Mathew PJ, Mathew JL (2003) Assessment and management of pain in infants. Postgrad Med J 79, 438-443.

McLeod GA, Burke D (2001) Levobupivacaine. Anaesthesia 56, 331-341.

Mercadante S, Arcuri E (2004) Opioids and renal function. J Pain 5, 2-19.

Monteiro ER, Junior AR, Assis HM et al. (2009) Comparative study on the sedative effects of morphine, methadone, butorphanol or tramadol, in combination with acepromazine, in dogs. Vet Anaesth Analg 36, 25-33.

Murrell JC, Psatha EP, Scott EM et al. (2008) Application of a modified form of the Glasgow pain scale in a veterinary teaching centre in the Netherlands. Vet Rec 162, 403-408.

Odette O, Smith LJ (2013) A comparison of epidural analgesia provided by bupivacaine alone, bupivacaine + morphine, or bupivacaine + dexmedetomidine for pelvic orthopedic surgery in dogs. Vet Anaesth Analg 40, 236-527.

Ranasinghe JS, Steadman J, Toyama T et al. (2003) Combined spinal epidural anaesthesia is better than spinal or epidural alone for Caesarian delivery. Br J Anaesth 91, 299-300. 
Rawal N, Mollefors K, Axelsson K et al. (1983) An experimental study of urodynamic effects of epidural morphine and naloxone reversal. Anaesth Analg 62, 641-647.

Reina MA, Franco CD, Lopéz A et al. (2009) Clinical implication of epidural fat in the spinal canal. A scanning electron microscopic study. Acta Anaesthesiol Belg 60, 7-17.

Torske KE, Dyson DH (2000) Epidural analgesia and anesthesia. Vet Clin North Am Small Anim Pract 30, 859-874.

Troncy E, Junot S, Keroak S et al. (2002) Results of preemptive epidural administration of morphine with or without bupivacaine in dogs and cats undergoing surgery: 265 cases (1997-1999). J Am Vet Med Assoc $221,666-672$.

Yeager MP, Glass DD, Neff RK et al. (1987) Epidural anesthesia and analgesia in high-risk surgical patients. Anesthesiology 66, 729-736.

Received 24 April 2015; accepted 29 January 2016.

Available online 11 January 2017

\section{Appendix A. Supplementary data}

Supplementary data related to this article can be found at http://dx.doi.org/10.1016/j.vaa.2016.01. 001. 